\title{
Quantitative magnetic resonance imaging in multiple sclerosis: the effect of high dose intravenous methylprednisolone
}

\author{
J KESSELRING,* D H MILLER, * D G MacMANUS, * G JOHNSON,* \\ N M MILLIGAN, $\uparrow$ N SCOLDING, $\uparrow$ D A S COMPSTON, $\uparrow$ W I McDONALD*
}

From the Multiple Sclerosis NMR Research Group, The National Hospital for Nervous Diseases, ${ }^{*}$ Queen Square, London, and the Section of Neurology, University of Wales, College of Medicine, Cardiff, UK

SUMMARY Magnetic resonance imaging was performed on 50 patients with clinically definite or probable multiple sclerosis before and 15 days after starting treatment with intravenous methylprednisolone ( $0.5 \mathrm{~g}$ daily for 5 days). Scans were abnormal in 49 patients. New lesions had appeared on the second scan in nine individuals and in seven a single pre-existing lesion appeared to have become smaller but in no case were lesions seen to disappear. Two patients showed both reduction in the size of an abnormal area and development of a single new lesion indicating that corticosteroids do not appear rapidly to alter the process underlying plaque formation. Measurements of relaxation times were performed in 12 randomly selected patients. All showed elevated values in normal appearing white matter but not cortex before treatment compared with 18 healthy controls. After treatment a significant decrease of $T_{1}$ and $T_{2}$ was observed in cortex, and of $T_{1}$ alone in normal appearing white matter. No significant change could be detected within lesions, a finding attributed to the wide range of relaxation values observed at these sites before treatment. Since brain water content is increased in normal appearing white matter of multiple sclerosis patients, and is significantly reduced by high dose methylprednisolone, resolution of oedema may contribute to the rapid spontaneous or corticosteroid induced symptomatic recovery that characterises the disease in its early stages.

Corticosteroids are widely used to treat acute exacerbations in patients with multiple sclerosis. ${ }^{1-3}$ Doubleblind controlled trials have demonstrated that corticotrophin or methylprednisolone are effective in reducing the duration of an attack but neither influences the long term course of the disease. ${ }^{45}$ It has been suggested that resolution of oedema, characteristic of the early lesion in multiple sclerosis, ${ }^{6}$ is the main mechanism underlying early symptomatic improvement. ${ }^{7}$ The rate of recovery induced by methylprednisolone is consistent with this hypothesis; experimentally resolution of oedema is associated with rapid restoration of function which can occur within a few days. ${ }^{8}$

Imaging appearances consistent with blood-brain barrier (BBB) damage are often seen in acute multiple

Address for reprint requests: Professor W I McDonald, Institute of Neurology, The National Hospital, Queen Square, London WCIN 3BG.

Received 10 May 1988 and in revised form 4 August 1988. Accepted 8 August 1988 sclerosis lesions ${ }^{9}$ and transient reversal of these abnormalities has been demonstrated within 8 hours of administering methylprednisolone; ${ }^{10}$ however the putative BBB abnormality may later reappear. "It has been shown that corticotrophin produces reversible brain shrinkage in children with infantile spasms, suggesting a dehydrating effect of corticosteroid therapy. ${ }^{12}$ There are thus several lines of evidence indicating that in multiple sclerosis corticosteroids might act by both reducing water content in the brain and affecting permeability of the BBB.

Proton magnetic resonance imaging (MRI) is highly sensitive in demonstrating the brain lesions in multiple sclerosis ${ }^{13}$ and provides a method for quantifying the disease process. Affected areas can be reliably distinguished from normal appearing white matter (NAWM), cortex and cerebrospinal fluid. The relaxation times $T_{1}$ and $T_{2}$ both positively correlate with the total amount of water and its physical state,$^{14}$ so that their measurement allows an assessment of the water content in normal and affected tissue.

Although MRI has an established role as an aid to 
clinical diagnosis in the management of patients with multiple sclerosis, potentially it has an even more important application as a sensitive method of assessing treatment; in addition MRI may help in understanding the mechanisms of action of therapeutic agents in multiple sclerosis.

We have therefore evaluated the effect of treatment using methylprednisolone on the MRI appearance in multiple sclerosis patients and correlated these with short term alterations in clinical disability.

\section{Methods}

Twenty three male and 27 female patients with clinically definite or probable multiple sclerosis according to the Poser criteria $^{15}$ aged 22 to 58 (mean 40.7) years, of whom eight were aged > 50 years, had MRI performed on a Picker 0.5 Tesla superconducting imager using the same sequences immediately before and 3-7 days after treatment with methylprednisolone $\left(0.5 \mathrm{~g}\right.$ Solu-Medrone ${ }^{\oplus}$ [Upjohn] dissolved in $7.8 \mathrm{ml}$ of benzyl alcohol $[0.9 \% \mathrm{w} / \mathrm{v}]$ given by slow intravenous infusion in $500 \mathrm{ml}$ of normal saline daily for 5 days as previously described). ${ }^{s}$ Informed consent was obtained from the patients and the protocol had been approved by the ethics committees.

The whole brain was examined initially with a 24 slice moderately $T_{2}$ weighted spin echo (SE) sequence $\left(\mathrm{SE}_{2000 / 60}\right.$; the first subscript refers to repetition time and the second to echo time). Slices were approximately contiguous and had a thickness of $5 \mathrm{~mm}$. The field of view was $30 \mathrm{~cm}$ with a $256 \times 256$ matrix and two averages. Accurate repositioning of the patient (to within $\pm 4^{\circ}$ angulation) was crucial for comparing the paired scans. The techniques by which this was achieved have previously been described. ${ }^{13}$ The MR images were reviewed by two observers (JK, DHM). The paired scans obtained from each individual were considered to differ if there was unequivocal evidence for one or more new lesions, and disappearance or definite increase/decrease in size of pre-existing abnormal areas. No significance was attached to slight variations in the size of a lesion which might have arisen from failure accurately to reposition the patient.

Measurements of relaxation times were performed on MRI scans of 12 randomly selected patients (8 female and 4 male) aged 30-56 (mean 44.4) years. Eighteen healthy controls were also studied. From the initial scan a small region containing well defined lesions, and areas of apparently unaffected cortex and white matter were selected for measurement of relaxation time. Three sets of images (two spin echoes ( $\mathrm{SE}_{2000 / / 00}$ and $\left.\mathrm{SE}_{2000 / 120}\right)$ and one inversion recovery $\left(\mathrm{IR}_{2000 / 40 / 500}\right.$ the first subscript refers to repetition time, the second to echo time and the third to inversion time)) were obtained from each region. Each set of images consisted of eight $5 \mathrm{~mm}$ thick slices. The image matrix was $128 \times 256$ over a $30 \mathrm{~cm}$ field of view and two signal averages were used. Relaxation time images were then calculated from these images. This method of analysis has been shown to be precise and stable over a period of 9 months. ${ }^{16}$

Relaxation times were measured in selected regions of interest (ROIs) within cortex, normal appearing white matter (NAWM), and lesions. In the cortex, relaxation times were measured within a ROI of $<0.1 \mathrm{~cm}^{2}$. In order to avoid artefactual high and low values caused by inclusion of cerebrospinal fluid or white matter within the cortical ROIs, the mean of three areas was taken. For each patient $T_{1}$ and $T_{2}$ relaxation times were measured in 30-36 different parts of the cerebral cortex. In NAWM values were measured within ROIs of $<0.1 \mathrm{~cm}^{2}$. To avoid inclusion of grey matter, measurements were taken at points where low readings had been recorded. $T_{1}$ and $T_{2}$ relaxation times were measured in 10-12 regions. Lesions were identified visually, selected for analysis if they were clearly identifiable in both scans and outlined using a tracking cursor; mean values with standard deviations were then obtained for $T_{1}$ and $T_{2}$. The highest value within each lesion was also recorded. Measurements were performed on pre and post-treatment scans by the same examiner (JK) without prior knowledge of their order. Measurements of $T_{1}$ and $T_{2}$ from the healthy normal controls have previously been reported. ${ }^{13}$ For statistical analysis, mean $T_{1}$ and $T_{2}$ values from the scans obtained before and after treatment were compared using a paired $t$ test for cortex, NAWM, and lesions separately.

\section{Results}

All patients were in a clinically stable phase or had slowly progressive disease; none had had a recent relapse. Forty-nine of 50 had abnormal neurological signs. At entry, all were fully ambulant although three required unilateral support when walking. Initially the Kurtzke expanded disability status scale (DSS) ${ }^{17}$ was $<4$ in $46 / 50$. After treatment scores were lower by $>0.5$ points on the DSS in 20 participants; 25 patients were unchanged and five had deteriorated by $>0.5$ points. One of these five individuals developed new sensory signs during treatment and relapsed symptomatically thereafter; four others developed new symptoms $2,3,8$, and 12 weeks after completing treatment. The DSS improved between the paired MRI scans in three patients and remained stable in the other.

Five of twelve patients, randomly selected for serial quantitative assessment of $T_{1}$ and $T_{2}$ relaxation times but clinically representative of the whole series, improved by $>0.5$ points on the DSS, two deteriorated, and five were unchanged.

There were three or more white matter lesions, consistent with the diagnosis of multiple sclerosis in the cerebral hemispheres of $48 / 50$ patients; all 48 had two or more periventricular abnormalities. The majority of patients had in excess of 20 lesions of various size in the cerebral hemispheres. Forty four of 48 patients had one or more lesions in the brain stem or cerebellum. One of the two remaining patients had a single lesion adjacent to the posterior body of the left lateral ventricle and another in the midbrain; she was classified as a clinically definite case. The other patient had a history of relapsing symptoms, consistent with widespread demyelination but no objective physical 
Table Relaxation times in the brain of multiple sclerosis patients before and after treatment with high dose methylprednisolone

\begin{tabular}{llll}
\hline Value & Mean $(S D)$ & Range & $p$ \\
\hline T1 Ctx pre & $598(27)$ & $546-638$ & $<0.0005$ \\
T1 Ctx post & $566(32)$ & $484-608$ & \\
T1 NAWM pre & $384(11)$ & $360-407$ & $<0.005$ \\
T1 NAWM post & $372(12)$ & $348-389$ & \\
T1 lesion pre & $600(225)$ & $157-848$ & n.s. \\
T1 lesion post & $578(227)$ & $133-885$ & \\
T2 Ctx pre & $101(7)$ & $92-114$ & $<0.005$ \\
T2 Ctx post & $96(7)$ & $88-107$ & \\
T2 NAWM pre & $73(4)$ & $67-82$ & n.s. \\
T2 NAWM post & $72(4)$ & $63-79$ & \\
T2 lesion pre & $169(37)$ & $104-241$ & n.s. \\
T2 lesion post & $171(35)$ & $105-246$ & \\
\hline
\end{tabular}

Abbreviations: SD: standard deviation; Ctx: cortex; pre: before treatment; post: after treatment; NAWM: normal appearing white matter; n.s.: not significant.

signs although cerebrospinal fluid had previously been shown to be abnormal; MRI was normal at the time of this study.

Follow-up MRI was performed a mean of 15 days after the first study. In 36 patients there was no detectable change in the scan appearances. In nine individuals a single small new lesion had appeared but in no case was this detected clinically and $7 / 9$ of these patients had a lower DSS after methylprednisolone compared with pre-treatment scores. These lesions were seen in the cerebral (7) or cerebellar (2) hemisphere white matter. No case showed unequivocal expansion of an old lesion. In seven cases a preexisting abnormality was smaller after treatment although no lesion had disappeared. In two patients there was both a reduction in size of a pre-existing lesion, and the development of a small new one. Overall there was no correlation between these MRI changes and clinical observations in individual patients.

\section{Relaxation times}

The results of measuring $T_{1}$ and $T_{2}$ relaxation times and their statistical analysis are given in the table. The pre-treatment values for $T_{1}$ and $T_{2}$ in NAWM but not cortical grey matter in patients with multiple sclerosis were significantly higher than corresponding results in the healthy controls. After treatment there was a highly significant fall in the values for $T_{1}$ and $T_{2}$ in cortex compared with pre-treatment results whereas in NAWM only the $T_{1}$ value was significantly reduced after treatment with methylprednisolone.

\section{Discussion}

This study demonstrates that, using high resolution imaging, there is no visible change in MRI appearance of the great majority of multiple sclerosis lesions in the cerebral hemispheres after treatment with high dose methylprednisolone. It is possible that more subtle changes in size or shape do occur which are beyond the resolution of our system; a $1 \mathrm{~mm}$ change in diameter would be difficult to detect. Our failure to demonstrate an appreciable change in the appearance of most lesions in the present study is not surprising. Many of the abnormal areas studied will have been chronic with established demyelination and fibrillary gliosis. The latter makes an important contribution to the abnormal signal ${ }^{1819}$ which is unlikely to be changed by methylprednisolone. Conversely, lesions depending more on recent changes in BBB permeability and oedema might be expected to resolve or become smaller with corticosteroids. Such lesions are however rather uncommon in patients during remissions [unpubished observation], and all patients in the present study were in a clinically stable phase of the disease.

A significant elevation of $T_{1}$ and to a lesser extent $T_{2}$ in NAWM from patients with multiple sclerosis compared with controls has been demonstrated. ${ }^{91320}$ Larsson $e t a l^{21}$ however did not find an elevation of $T_{1}$ though they used different field strength (1.5 T), and their long term reproducibility in phantoms $(7 \%)$ was significantly worse than ours (3\%). It is highly likely that this finding results from increased water content already known to be present in the brain of multiple sclerosis patients. ${ }^{22}$ Pathological abnormalities have been described in macroscopically normal appearing white matter in the form of perivascular oedema or inflammation and astrocyte proliferation ${ }^{23}$ each of which will contribute to increased water content. The present study demonstrates that pulsed high dose intravenous methylprednisolone given to patients with multiple sclerosis significantly reduces $T_{1}$ in cortex and NAWM, and $T_{2}$ in cortex, measured 7 days after treatment. $T_{1}$ is largely dependent on total water content and even small changes in this will be readily detected. Conversely, $T_{2}$ is heavily influenced by the macromolecular constituents of the tissue being imaged, ${ }^{14}$ and small changes in water content may be less easy to identify. The changes we observed in the cortex may simply reflect the fact that this part of the cerebrum normally has a much higher water content than white matter and in this respect does not differ significantly from the healthy brain. ${ }^{13}$ But the highly significant reduction of $T_{1}$ and $T_{2}$ in grey matter produced by methylprednisolone indicates that in patients with multiple sclerosis corticosteroids reduced water content not only in oedematous NAWM but also in previously apparently normal brain tissue.

The mode of action of corticosteroids in improving function in the central nervous system is unknown although dispersal of oedema is an obvious possibility. ${ }^{710}$ Our observations are consistent with this

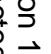


interpretation but do not preclude other or complementary effects including modification of local and systemic immunological processes ${ }^{24}$ or a direct effect on the development of demyelination..$^{25}$ It is unknown whether corticosteroids have a direct effect on electrophysiological properties of partially demyelinated central axons. It is perhaps surprising that we observed no apparent change in the relaxation times in the lesions themselves; we cannot at present determine whether this reflects an alteration in the properties of the BBB in chronic lesions rendering them insusceptible to the action of corticosteroids. However, the range of values obtained within lesions is so much wider than for NAWM, probably reflecting different stages in their evolution [unpublished observation], that even a change of the same order as that seen in NAWM could not reliably be detected.

This investigation further demonstrates the dissociation between disease activity and its clinical expression in patients with multiple sclerosis. Nine new lesions appeared on MRI which were all clinically silent, and the reduction in size of other abnormal areas was not detected clinically. Although not a primary aim of the study, our observations also illustrate the therapeutic limitations of high dose intravenous methylprednisolone in patients with multiple sclerosis and show that by contrast with individuals in acute relapse, or those with progressive disease affecting the pyramidal system, patients with fixed but stable clinical deficit do not benefit usefully from this treatment; nor do corticosteroids prevent the appearance of new lesions even in the short term. This study does, however, demonstrate for the first time the value of MRI in assessing mechanisms of action of a putative new treatment for patients with multiple sclerosis and perhaps other diseases of the central nervous system.

The Picker NMR machine used in this study was generously provided by the Multiple Sclerosis Society of Great Britain and Northern Ireland. JK was supported by a grant from the Schweizerische Stiftung für medizinisch-biologische Stipendien, DHM, DGMacM and GJ by grants from the Medical Research Council.

\section{References}

1 Therapeutic Claims in Multiple Sclerosis. International Federation of Multiple Sclerosis Societies, 2nd ed. London: Macmillan, 1988.

2 Dowling PC, Bosch VV, Cook SD. Possible beneficial effect of high dose intravenous steroid therapy in acute demyelinating disease and transverse myelitis. Neurology 1980;30:33-6.

3 Barnes MP, Bateman DE, Cleland PG, et al. Intravenous methylprednisolone for multiple sclerosis in relapse. $J$ Neurol Neurosurg Psychiatry 1985;48:157-9.

4 Rose AS, Kuzma JW, Kurtzke JF, Namerow NS, Sibley WA, Tourtellotte WW. Cooperative study in the evaluation of multiple sclerosis: ACTH vs. placebo. Final report. Neurology 1970;20:1-59.

5 Milligan NM, Newcombe R, Compston DAS. A double-blind controlled trial of high dose methylprednisolone in patients with multiple sclerosis. 1. Clinical effects. J Neurol Neurosurg Psychiatry 1987;50:511-6.

6 Silberberg DH. Pathogenesis of demyelination. In: McDonald WI, Silberberg DH, eds. Multiple Sclerosis. London: Butterworths 1986:99-111.

7 Halliday AM, McDonald WI. Pathophysiology of MS. Br Med Bull 1977;33:21-7.

8 Jacobson SG, Eames RA, McDonald WI. Optic nerve fibre lesions in adult cats: pattern of recovery of spatial vision. Exp Brain Res 1979;36:491-508.

9 Miller DH, Rudge P, Johnson G, et al. Serial gadolinium enhanced magnetic resonance imaging in multiple sclerosis. Brain 1988; (in press).

10 Troiano R, Hafstein M, Ruderman M, Dowling P, Cook S. Effect of high-dose intravenous steroid administration on contrastenhancing computed tomography scan lesions in multiple sclerosis. Ann Neurol 1984;15:257-63.

11 Lodder J, de Weerd AW, Koetsier JC, van der Lugt PJM. Computed tomography in acute cerebral multiple sclerosis. A report of two cases. Arch Neurol 1985;40:320-2.

12 Lagenstein I, Willig RP, Kühne D. Cranial computed tomography (CCT) findings in children treated with ACTH and dexamethasone: first results. Neuropädiatrie 1979;10:370-84.

13 Ormerod IEC, Miller DH, McDonald WI, et al. The role of NMR imaging in the assessment of multiple sclerosis and isolated neurological lesions: a quantitative study. Brain 1987;110: $1579-616$.

14 Mathur-De Vré R. Biomedical implications of the relaxation behaviour of water related to NMR imaging. $B r J$ Radiol 1984;57:955-76.

15 Poser CM, Paty DW, Scheinberg LC, et al. New diagnostic criteria for multiple sclerosis: guidelines for research protocols. Ann Neurol 1983;13:227-31.

16 Johnson G, Ormerod IEC, Barnes D, Tofts PS, MacManus D. Accuracy and precision in the measurement of relaxation times from nuclear magnetic resonance images. $\mathrm{Br} J$ Radiol 1987;60:143-53.

17 Kurtze JF. Rating neurological impairment in multiple sclerosis: An expanded disability status scale (DSS). Neurology 1983;33:1444-52.

18 McDonald WI. Gowers lecture: the mystery of the origin of multiple sclerosis. J Neurol Neurosurg Psychiatry 1986;49: 113-23.

19 Barnes D, McDonald WI, Landon DN, Johnson G. The characterisation of experimental gliosis by quantitative nuclear magnetic resonance imaging. Brain 1988;111:83-94.

20 Lacomis D, Osbakken M, Gross G. Spin-lattice relaxation ( $\left.T_{1}\right)$ times of cerebral white matter in multiple sclerosis. Mag Res Med 1986;3:194-202.

21 Larrson HBW, Frederiksen J, Kjaer L, Henriksen O, Oleson J. In vivo determination of $T_{1}$ and $T_{2}$ in the brain of patients with severe but stable multiple sclerosis. Magn Reson Med 1988;7: 43-55.

22 Tourtellotte WW, Parker JA. Some spaces and barriers in postmortem multiple sclerosis. In: Lajtha A, Ford DH (eds): Brain Barrier Systems, Progress in Brain Research 1968;29: 493-525.

23 Allen IV, McKeown SR. A histological, histochemical, and biochemical study of the macroscopically normal appearing white matter in multiple sclerosis. J Neurol Sci 1979;41:81-91.

24 Compston DAS, Milligan NM, Hughes PJ, et al. A double-blind controlled trial of high dose methylprednisolone in patients with multiple sclerosis: 2. Laboratory results. J Neurol Neurosurg Psychiatry 1987;50:517-22.

25 Triarhou LC, Herndon RM. The effect of dexamethasone on L-alpha-lysophosphatidylcholine (lysolecithin)-induced demyelination of the rat spinal cord. Arch Neurol 1986;43: 121-5. 